\title{
Sharp energy estimates for finite element approximations of non-convex problems
}

Chipot, M ; Müller, S

\begin{abstract}
From the introduction: The goal of this note is to expose in a simple situation the key arguments which allow one to prove sharp energy estimates in the numerical analysis of problems with multiple-well energy in the calculus of variations. Let us recall that such problems arise naturally for instance in materials science.
\end{abstract}

Other titles: Proceedings of the IUTAM Symposium held at the École des Mines and the École Polytechnique, Paris, April 22-25, 1997

Posted at the Zurich Open Repository and Archive, University of Zurich ZORA URL: https://doi.org/10.5167/uzh-22134

Book Section

Originally published at:

Chipot, M; Müller, S (1999). Sharp energy estimates for finite element approximations of non-convex problems. In: Argoul, P; Frémond, M; Nguyen, Q S. Variations of domain and free-boundary problems in solid mechanics (Paris, 1997). Dordrecht: Kluwer Academic, 317-325. 


\section{Max-Planck-Institut für Mathematik \\ in den Naturwissenschaften \\ Leipzig}

Sharp energy estimates for finite element approximations of non-convex problems

by

M. Chipot and S. Müller

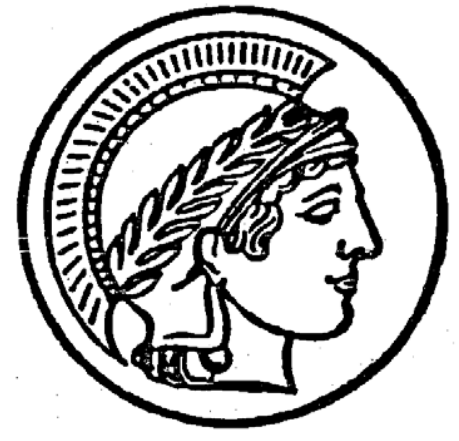





\title{
SHARP ENERGY ESTIMATES FOR FINITE ELEMENT APPROXIMATIONS OF NON-CONVEX PROBLEMS
}

\author{
M. CHIPOT \\ Mathematische Institut, Universität Zürich \\ Winterthurstr. 190 - 8057 Zürich - Switzerland \\ AND \\ S. MÜLLER \\ MPI für Mathematik in den Naturwissenschaften \\ Inselstr. 22-26 - 04103 Leipzig - Germany
}

\section{Introduction}

The goal of this note is to expose in a simple situation the key arguments which allow one to prove sharp energy estimates in the numerical analysis of problems with multiple-well energy in the calculus of variations. Let us recall that such problems arise naturally for instance in materials science. We refer the reader for this matter to [1], [5], [6], [7], [10]. Note also that our technique borrows widely from [8], [9] and can be extended to more general situations - see [3].

So, we will assume that we are in dimension 2 and $\Omega$ will denote the square $(0,1) \times(0,1)$ with boundary $\partial \Omega$. Setting

$$
W_{0}^{1, \infty}(\Omega)=\{v: \Omega \rightarrow \mathbf{R} \mid v \text { is Lipschitz continuous, } v=0 \text { on } \partial \Omega\}
$$

we would like to consider the problem

$$
\inf _{W_{0}^{1, \infty}(\Omega)} \int_{\Omega} W(\nabla u) d x d y=\inf _{W_{0}^{1, \infty}(\Omega)} \int_{\Omega}\left|u_{x}\right|+|| u_{y}|-1| d x d y
$$

where we have set

$$
\nabla u=\left(u_{x}, u_{y}\right) \quad, \quad W\left(\xi_{1}, \xi_{2}\right)=\left|\xi_{1}\right|+|| \xi_{2}|-1| .
$$

More precisely we would like to address a discrete version of this problem. We denote by $\tau_{h}$ a regular (see [4]) family of triangulations of $\Omega$, with mesh 
size $h$, i.e.

$$
h=\max \left\{\operatorname{diam}(\mathrm{K}) \mid \mathrm{K} \in \tau_{\mathrm{h}}\right\} .
$$

Then, if $P_{1}$ denotes the set of polynomials of degree one, we set

$$
V_{0}^{h}=\left\{v: \Omega \rightarrow \mathbf{R} \mid v \text { continuous, }\left.v\right|_{k} \in P_{1} \forall k \in \tau_{h}, v=0 \text { on } \partial \Omega\right\}
$$

and we consider the discretized analogue of (1.1) :

$$
\inf _{V_{0}^{h}} \int_{\Omega} W(\nabla u) d x d y
$$

The energy density $W$ is a nonnegative function that vanishes only on the two wells

$$
\omega_{+}=(0,1) \quad, \quad \omega_{-}=(0,-1) .
$$

They play, of course, a crucial role in the minimization process. It is easy to establish that (1.1) does not admit a minimizer but that (1.3) does (see for instance [2]) thus the minimizers of (1.3) provide a minimizing sequence to (1.1). What we would like to establish here is:

Theorem 1 There exists a constant $C$ independent of $h \ll 1$ such that

$$
\inf _{V_{0}^{h}} \int_{\Omega}\left|u_{x}\right|+|| u_{y}|-1| d x d y \leq C h^{\frac{1}{2}} .
$$

Moreover, this estimate is sharp in the sense that there exists a family of triangulation $\tau_{h}$, a constant $c$ independent of $h$ such that

$$
\inf _{V_{0}^{h}} \int_{\Omega}\left|u_{x}\right|+|| u_{y}|-1| d x d y \geq c h^{\frac{1}{2}} .
$$

The rest of the paper will be devoted to the proof of this theorem.

\section{Proof of Theorem 1}

\subsection{THE PROOF OF (1.5)}

Let us denote by $\alpha \in(0,1)$ a real number that we will fix later on. Define

$$
u(x, y)= \begin{cases}y & \text { if } \quad y \in\left[0, h^{\alpha}\right] \\ 2 h^{\alpha}-y & \text { if } \quad y \in\left[h^{\alpha}, 2 h^{\alpha}\right] .\end{cases}
$$

Assume that $u$ is extended periodically - with period $2 h^{\alpha}$ in the $y$ direction - on the whole $\mathbf{R}^{2}$. Clearly one has

$$
\nabla u=\omega_{+} \quad \text { or } \omega_{-} \quad \text { a.e. } \quad \text { in } \Omega .
$$


Since $u$ does not vanish on the boundary of $\Omega$ one sets

$$
\hat{u}=u \wedge \operatorname{dist}(x, \partial \Omega)
$$

and then

$$
u_{h}=\text { the interpolate of } \hat{u} \text { on } \tau_{h},
$$

i.e. $u_{h}$ denotes the function of $V_{0}^{h}$ that agrees with $\hat{u}$ at the nodes of the triangulation $\tau_{h}$. The two functions that are involved in the definition of $\hat{u}$ have a gradient that is uniformly bounded. Since the triangulation $\tau_{h}$ is regular, the gradient of $u_{h}$ is uniformly bounded independently of $h$ (see [4]). It then follows from (2.8) that one has

$$
\int_{\Omega} W\left(\nabla u_{h}\right) d x d y=\int_{\left\{u_{h} \neq u\right\}} W\left(\nabla u_{h}\right) d x d y \leq C\left|\left\{u_{h} \neq u\right\}\right|
$$

for some constant $C$, where $\left|\left\{u_{h} \neq u\right\}\right|$ denotes the measure of the set where $u_{h}$ is distinct from $u$. First one remarks that

$$
0 \leq u \leq h^{\alpha}
$$

(see (2.7)) and thus for $\operatorname{dist}(x, \partial \Omega)>h^{\alpha}$ one has $\hat{u}=u$. So, for $\operatorname{dist}(x, \partial \Omega) \geq$ $h^{\alpha}+h$ the interpolate of $\hat{u}$ will be equal to the interpolate of $u$. Now, the interpolate of $u$ is $u$ itself except on a strip of size $2 h$ around each of the lines $y=k h^{\alpha}, k \in \mathbf{N}$. Collecting this information one has clearly

$$
\left|\left\{u_{h} \neq u\right\}\right| \leq 4\left(h^{\alpha}+h\right)+N 2 h \leq 8 h^{\alpha}+(N+1) 2 h
$$

where $N$ is the number of strips cutting $\Omega$. Note that for $h<1$ and $\alpha \in$ $(0,1)$ one has $h<h^{\alpha}$. Now, one has clearly

$$
(N+1) h^{\alpha} \leq 1
$$

so that by $(2.10),(2.11)$ one gets

$$
\int_{\Omega} W\left(\nabla u_{h}\right) d x d y \leq 8 C\left(h^{\alpha}+h^{1-\alpha}\right) .
$$

Taking $\alpha=\frac{1}{2}$ leads to (1.5).

\subsection{PROOF OF (1.6)}

First remark that if we claim that (1.5) is sharp there are some particular triangulations - related to the wells - for which (1.5) could be improved. Indeed, choose for instance the triangulation of the (figure 1) and set 
1

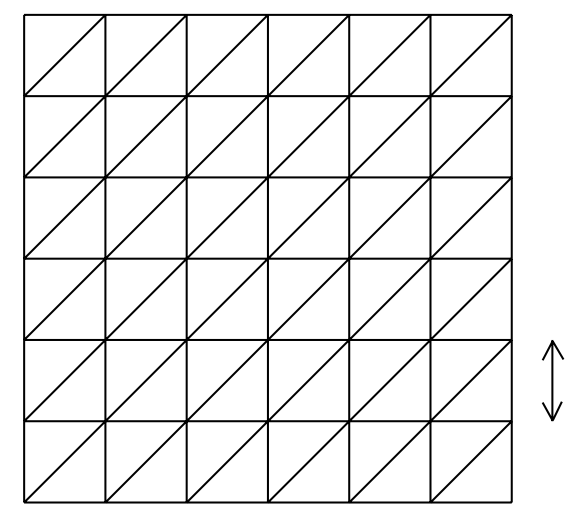

$$
h^{\prime}=h / \sqrt{2}
$$

0

Figure 1.

$$
u(x, y)= \begin{cases}y & \text { on }\left(0, h^{\prime}\right) \\ 2 h^{\prime}-y & \text { on }\left(h^{\prime}, 2 h^{\prime}\right) .\end{cases}
$$

Extending $u$ periodically with $2 h^{\prime}$, and setting

$$
u_{h}=\text { the interpolate of } u \wedge \operatorname{dist}(x, \partial \Omega)
$$

one clearly has

$$
\inf _{V_{0}^{h}} \int_{\Omega} W(\nabla u) d x d y \leq \int_{\Omega} W\left(\nabla u_{h}\right) d x d y \leq c h \ll \operatorname{ch}^{\frac{1}{2}}
$$

for $h$ small. However, this estimate depends on the triangulation as we are about to see. Indeed consider now a family of triangulations as on (figure 2). For $x_{0}=k h / 2, k \in \mathbf{N}$ we are going to consider the $\operatorname{strip}\left(x_{0}, x_{0}+\frac{h}{2}\right) \times(0,1)$. Let $u \in V_{0}^{h}$ and $0<\delta<\frac{1}{3}$. Recall that $\nabla u$ is constant on each of the triangles of $\tau_{h}$. Then for a given $u$ let us adopt the following definition:

Definition 1 Let $T \in \tau_{h}$ with one side at least of length $h$. We will say that $T$ is of

$$
\begin{array}{llll}
\text { type }+ & \text { if } & \left|u_{x}\right| \leq \delta, & \left|u_{y}-1\right| \leq \delta, \\
\text { type }- & \text { if } & \left|u_{x}\right| \leq \delta, & \left|u_{y}+1\right| \leq \delta, \\
\text { type } 0 & \text { else. } & &
\end{array}
$$

In other words $T$ is of type + or - if $\nabla u$ is close to $\omega_{+}$or $\omega_{-}$respectively. Then on the strip $\left(x_{0}, x_{0}+\frac{h}{2}\right) \times(0,1)$ we have triangles of various types. We denote by $N_{0}=N_{0}\left(x_{0}\right)$ the number of triangles of type 0 . We say that we have a change of phase along the line $x_{0}$ when two triangles change from type + to type - (see figure 3 ). Then we can prove, 
1

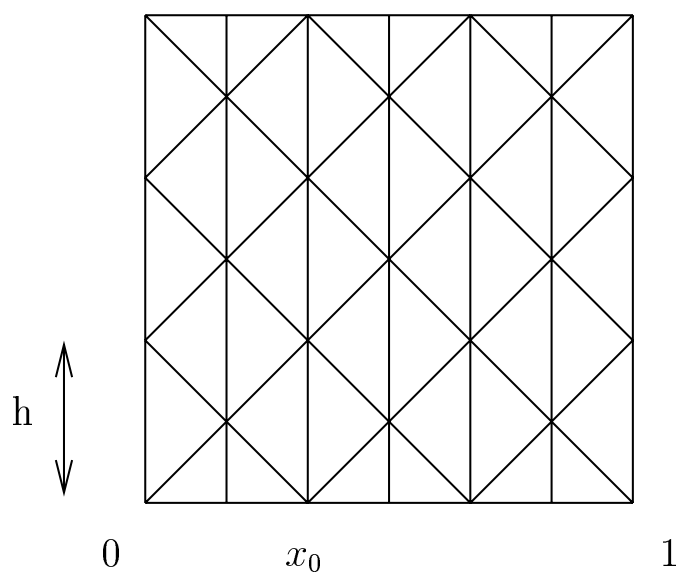

Figure 2.

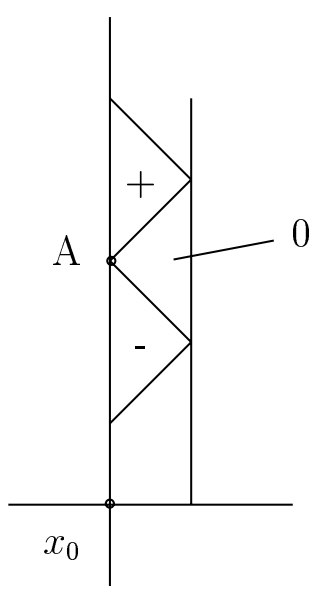

Figure 3.

Lemma 1 At each change of phase along the line $x=x_{0}$ the triangle having only one vertex on $x=x_{0}$ is of type 0 , (see figure 3).

Proof. Let us denote by $\left(u_{x}^{+}, u_{y}^{+}\right),\left(u_{x}^{-}, u_{y}^{-}\right)$the gradients of $u$ in the triangle of type + and of type - , respectively. If $A=\left(x_{0}, y_{0}\right)$ one has

$$
u\left(x_{0}+\frac{h}{2}, y_{0}+\frac{h}{2}\right)=u\left(x_{0}, y_{0}\right)+u_{x}^{+} \frac{h}{2}+u_{y}^{+} \frac{h}{2}
$$




$$
u\left(x_{0}+\frac{h}{2}, y_{0}-\frac{h}{2}\right)=u\left(x_{0}, y_{0}\right)+u_{x}^{-} \frac{h}{2}-u_{y}^{-} \frac{h}{2} .
$$

So, in the triangle whose only vertex on $x=x_{0}$ is $A$ one has

$$
u_{y}=\frac{1}{2}\left(u_{x}^{+}-u_{x}^{-}\right)+\frac{1}{2}\left(u_{y}^{+}+u_{y}^{-}\right) .
$$

Since $u_{x}^{+}, u_{x}^{-} \in[-\delta, \delta], \quad u_{y}^{+} \in[1-\delta, 1+\delta], \quad u_{y}^{-} \in[-1-\delta,-1+\delta]$ one deduces that $\left|u_{y}\right| \leq 2 \delta$ so that

$$
\left|u_{y} \pm 1\right| \geq 1-2 \delta>\delta
$$

Since $0<\delta<\frac{1}{3}$. The considered triangle is thus of type 0 .

Lemma 2 Let $u$ be a piecewise $\mathbf{C}^{1}$ function on $(a, b)$ such that $u^{\prime}$ has a constant sign and $\left|u^{\prime}\right| \geq C$. Then one has

$$
\int_{a}^{b}|u(z)| d z \geq C\left(\frac{(b-a)^{2}}{4}\right)
$$

Proof. If $u$ does not vanish on $[a, b]$ then clearly

$$
\int_{a}^{b}|u(z)| d z \geq \min \left(\int_{a}^{b}|u(z)-u(a)| d z, \int_{a}^{b}|u(z)-u(b)| d z\right)
$$

So, considering possibly $u-u(a)$ or $u-u(b)$ instead of $u$ one can assume that $u$ vanishes at some point $\xi$. Since $u^{\prime}$ has a constant sign one has

$$
|u(z)|=\left|u(\xi)+\int_{\xi}^{z} u^{\prime}\left(z^{\prime}\right) d z^{\prime}\right|=\int_{[\xi, z]}\left|u^{\prime}(z)\right| d z \geq C|\xi-z| .
$$

Integration in $z$ on $(a, b)$ leads to $(2.12)$.

Lemma 3 Assume that $\left(N_{0}+1\right) h \leq \frac{1}{2}$ then one has

$$
\int_{0}^{1}\left|u\left(x_{0}, y\right)\right| d y \geq \frac{1-\delta}{16\left(N_{0}+1\right)} .
$$

Proof. Consider a maximal chain of triangles of type + or - . In other words consider $\left(a_{i}, b_{i}\right)$ such that one has a change of phase or a "boundary point" at $\left(x_{0}, a_{i}\right),\left(x_{0}, b_{i}\right)$ and all the sides of triangles of the strip $\left(x_{0}, x_{0}+\frac{h}{2}\right) \times(0,1)$ located on $x=x_{0}$ between $a_{i}$ and $b_{i}$ belong to triangles of the same type + or -. (A "boundary point" could be a vertex of one triangle of the boundary 
with all its sides strictly smaller than $\mathrm{h}$.) Since $u_{y}$ has a constant sign and $\left|u_{y}\right| \geq 1-\delta$ one deduces from Lemma (2) that

$$
\int_{a_{i}}^{b_{i}}\left|u\left(x_{0}, y\right)\right| d y \geq(1-\delta) \frac{\left(b_{i}-a_{1}\right)^{2}}{4} .
$$

Denote by $N_{1}$ the number of maximal chains as above, i.e. $i=1, \ldots, N_{1}$. Then from (2.14) one deduces

$$
\sum_{i=1}^{N_{1}} \int_{a_{i}}^{b_{i}}\left|u\left(x_{0}, y\right)\right| d y \geq \frac{(1-\delta) N_{1}}{4} \sum_{i=1}^{N_{1}} \frac{\left(b_{i}-a_{i}\right)^{2}}{N_{1}}
$$

and by a convexity argument

$$
\int_{0}^{1}\left|u\left(x_{0}, y\right)\right| d y \geq \sum_{i=1}^{N_{1}} \int_{a_{i}}^{b_{i}}\left|u\left(x_{0}, y\right)\right| d y \geq \frac{(1-\delta) N_{1}}{4}\left(\sum_{i=1}^{N_{1}} \frac{b_{i}-a_{i}}{N_{1}}\right)^{2} .
$$

Due to Lemma 1 the sides of triangles on $x=x_{0}$ which do not belong to a chain belong to a triangle of type 0 or a small triangle of the boundary. So, one has

$$
\sum_{i=1}^{N_{1}} b_{i}-a_{i} \geq 1-\left(N_{0}+1\right) h \geq \frac{1}{2}
$$

and from (2.15) we deduce that

$$
\int_{0}^{1}\left|u\left(x_{0}, y\right)\right| d y \geq \frac{(1-\delta)}{16 N_{1}} .
$$

The result follows from the fact that by Lemma $1, N_{1} \leq N_{0}+1$.

Lemma 4 Let $u \in V_{0}^{h}$ then one has

$$
\int_{0}^{1}\left|u\left(x_{0}, y\right)\right| d y \leq \int_{\Omega} W(\nabla u) d x d y .
$$

Proof. One has

$$
u\left(x_{0}, y\right)=u(0, y)+\int_{0}^{x_{0}} u_{x}(\xi, y) d \xi=\int_{0}^{x_{0}} u_{x}(\xi, y) d y
$$

Thus

$$
\left|u\left(x_{0}, y\right)\right| \leq \int_{0}^{1}\left|u_{x}(\xi, y)\right| d \xi \leq \int_{0}^{1} W(\nabla u(\xi, y)) d \xi
$$

and the result follows by integration in $y$. 
Lemma 5 (Estimate of the number of changes of phase). Let $u \in V_{0}^{h}$ such that

$$
\int_{\Omega} W(\nabla u) d x d y \leq C h^{\frac{1}{2}}
$$

where $C$ is a constant. If $h \leq 64 C^{2}$ then one has

$$
N_{0}+1 \geq \frac{1-\delta}{16 C} h^{-\frac{1}{2}}
$$

(Recall that $N_{0}$ is the number of triangles of type 0 in the strip $\left(x_{0}, x_{0}+\right.$ $\left.\frac{h}{2}\right) \times(0,1)$.)

Proof. If (2.18) does not hold one has

$$
\left(N_{0}+1\right) h<\frac{(1-\delta) h^{\frac{1}{2}}}{16 C}<\frac{h^{\frac{1}{2}}}{16 C} \leq \frac{1}{2} .
$$

Thus combining (2.13),(2.16) and (2.17) one obtains

$$
\frac{1-\delta}{16\left(N_{0}+1\right)} \leq \int_{0}^{1}\left|u\left(x_{0}, y\right)\right| d y \leq C h^{\frac{1}{2}}
$$

which leads to (2.18).

\section{End of the proof of Theorem 1}

Knowing that (1.5) holds consider a $u \in V_{0}^{h}$ such that

$$
\int_{\Omega} W(\nabla u) d x d y \leq C h^{\frac{1}{2}}
$$

On a triangle of type 0 for such an $u$ one has

$$
\left|u_{x}\right| \geq \delta \text { or }|| u_{y}-1 \mid \geq \delta
$$

i.e. $W(\nabla u) \geq \delta$. Thus if $S_{x_{0}}=\left(x_{0}, x_{0}+\frac{h}{2}\right) \times(0,1)$

$$
\int_{S_{x_{0}}} W(\nabla u) d x d y \geq N_{0} \delta \frac{h^{2}}{2} .
$$

Assuming that $h$ is small - more precisely $\frac{(1-\delta) h^{-\frac{1}{2}}}{32 C}>1$ - one deduces from (2.18)

$$
N_{0} \geq \frac{1-\delta}{32 C} h^{-\frac{1}{2}}
$$


which leads to

$$
\int_{S_{x_{0}}} W(\nabla u) d x d y \geq \frac{(1-\delta) \delta h^{\frac{3}{2}}}{64 C}
$$

Since the number of stripes $S_{x_{0}}$ is $\frac{2}{h}$ one deduces that

$$
\int_{\Omega} W(\nabla u) d x d y \geq \frac{(1-\delta) \delta h^{\frac{1}{2}}}{32 C}
$$

which completes the proof.

Remark. The exponent $1 / 2$ appearing in (1.6), (1.5) is related to the linear growth of $W$. Other growth conditions would produce other exponents in (1.6), (1.5). Our argument extend also to general polygonal domains and more general functionals. For all this we refer to [3].

Acknowledgements: This paper was written when the first author was visiting the Universidade de Beira Interior in Covilhã. He would like to thank the mathematics department for its support. The second author would like also to thank the mathematics department in the University of Metz for its hospitality at the early stage of this research.

\section{References}

1. J.M. Ball and R.D. James, Proposed experimental tests of a theory of fine microstructure and the two well problem, Phil. Trans. R. Soc. London A, 338, (1992) $389-450$.

2. M. Chipot, Numerical analysis of oscillations in nonconvex problems, Numerische Mathematik, 59, (1991), 747-767.

3. M. Chipot and S. Müller, In preparation.

4. Ph. Ciarlet, The finite element method for elliptic problems. North-Holland, Amsterdam (1978).

5. J. Ericksen, Constitutive theory for some constrained elastic crystal, Int. J. Solids and Structures, 22 (1986), 951-964.

6. R. James and D. Kinderlehrer, Theory of diffusionless phase transitions, Lecture Notes in Physics, 344, Springer Verlag (M. Rascle, D. Serre and M. Slemrod, Eds.) (1989), 51-84.

7. R. Kohn, Relaxation of a double-well energy, Continuum Mech. Thermodyn., 3 (1991), 193-236.

8. R. Kohn and S. Müller, Branching of twins near an austenite-martensite interface, Phil. Mag. A, 66 (1992), 697-715.

9. R. Kohn and S. Müller, Surface energy and microstructure in coherent phase transitions, CPAM, 47 (1994), 405-435.

10. M. Luskin, On the computation of crystalline microstructure, Acta Numerica, (1996), 191-257. 